\title{
Gerade jetzt! - \\ 10 Thesen, warum der Religionsunterricht in der Corona-Zeit unverzichtbar ist
}

https://doi.org/10.1515/zpt-2020-9090

Religion - wozu soll das jetzt gut sein? So oder ähnlich scheinen viele für die öffentliche Schulbildung Verantwortliche gedacht zu haben, als es darum ging, schulische Antworten auf die durch die Corona-Krise notwendig gewordenen Schutzmaßnahmen zu finden. Rasch und viel zu selbstverständlich wurde von Seiten der Ministerien und Schulleitungen eine Scheidelinie gesetzt - zwischen Kernfächern, auf die sich der reduzierte Schulbetrieb konzentrieren sollte, und Randfächern, die man entbehren kann, wenn es hart auf hart kommt. Folglich kamen letztere in den Monaten der Corona-Beschulung kaum mehr zum Zuge auch der Religionsunterricht nicht, um den es hier geht.

Die folgenden Thesen legen dar, weshalb der Religionsunterricht in der Corona-Zeit am Ort der Schule unverzichtbar ist. Sie entspringen nicht der defensiven Absicht, das Existenzrecht eines Schulfaches in Zeiten akuter Gefährdung zu behaupten. Vielmehr geht es hier ums Ganze: das Recht der Kinder und Jugendlichen auf umfassende Bildung. In Zeiten grundstürzender existenzieller Erschütterungen und eines entsprechend gestiegenen Orientierungsbedarfs ist eine solche weit gefasste Bildung wichtiger denn je. Daher gilt: Gerade jetzt darf der Religionsunterricht nicht ausfallen!

1. Schülerinnen und Schüler sowie Lehrkräfte haben in der Zeit der Schulschließungen und des Lockdown tiefgreifende Erfahrungen gemacht, die dringend thematisiert werden müssen. Erste wissenschaftliche Studien und Berichte machen deutlich, dass die Zeit von Homeschooling, Kontaktsperre oder auch behördlich angeordneter Quarantäne für viele Kinder und Jugendliche mit erheblichen Konsequenzen, etwa massiver sozialer Isolation, verbunden waren. In manchen Familien kam es zu akuten Erkrankungen und der Verlust nahestehender Menschen stand als Möglichkeit immer vor Augen bzw. hat sich in manchen Fällen als traurige Wirklichkeit eingestellt. Das Leben auf engem Raum führte zu Konflikten und Aggressionen, mitunter auch zu häuslicher Gewalt. Kinder und Jugendliche waren dabei ebenso Zuschauer und Zeugen wie auch selbst Opfer. 
Der Religionsunterricht bietet einen wichtigen Raum, in dem die existenziellen Erfahrungen der Corona-Krise gemeinsam zur Sprache gebracht und lebensrelevant bearbeitet werden.

2. In der Corona-Krise treten die Verletzlichkeit und Unverfügbarkeit des menschlichen Lebens verdichtet vor Augen. Religion als eine Kultur des Umgangs mit Unverfügbarem stellt eine Form von Weltzugang dar, die auf diese Erfahrungen reagiert und ihnen mit einer eigenen Ausdruckskultur begegnet. Religiöse Bildung leistet damit einen nicht ersetzbaren Beitrag zur allgemeinen Bildung. Trotzdem gehörte der Religionsunterricht in der Regel nicht zu den Fächern, die in das Bemühen um digitale Angebote aufgenommen wurden. Eine Reduktion von Schule auf sogenannte Kernfächer übersieht, dass eine wesentliche Grundaufgabe der Schule darin besteht, Kindern und Jugendlichen möglichst weitreichende Orientierungen zu ermöglichen. Was hier zu priorisieren ist, muss angesichts der existenziellen Krisenerfahrungen intensiv diskutiert werden.

Der Religionsunterricht ist an vielen Schulen geradezu ersatzlos gestrichen worden. Schulische Bildung kann sich angesichts der gegenwärtigen Krisenerfahrungen jedoch nicht auf die sogenannten Hauptfächer konzentrieren, wenn sie dem Anspruch umfassender Bildung auch in Krisenzeiten gerecht werden will.

3. Die Corona-Krise fordert eine verantwortliche Rede von Gott besonders heraus - und berührt nicht zuletzt auch unmittelbar Glaubensfragen. Warum ist es so weit gekommen? In was für einer Welt leben wir? Wie weit reicht der menschliche Anspruch auf Beherrschung und Beherrschbarkeit aller Probleme? Wie verhält sich die Rede von Gott zu den Leiderfahrungen der Menschen? Ist ein Strafhandeln Gottes vorstellbar? Dies macht theologische Deutungen der Würde jedes Lebens, des Umgangs mit unhintergehbaren Dilemmasituationen und (notwendiger) Schuld in unentscheidbaren Entscheidungssituationen sowie christliche Lebensdeutungen und -formen im Umgang mit Angst, Einsamkeit, Tod und Trauer erforderlich.

Der Religionsunterricht zielt darauf ab, angesichts der aktuellen Anforderungen biblisch-christliche Traditionen erfahrungs- und biographiebezogen ins Gespräch zu bringen. 
4. Die Krise drängt zur Deutung. Dabei erfahren Deutungsbedürftigkeit und Deutungsproduktion eine starke mediale Beschleunigung. Wer an der Aushandlung dessen, was als systemrelevant gelten soll und was nicht, beteiligt ist und wer nicht, verbindet sich mit Fragen nach Deutungsmacht. So haben sich auch extreme Positionen wie Verschwörungstheorien verschiedenster Art Gehör und Ausdruck in Demonstrationen verschafft.

Der Religionsunterricht zeichnet sich dadurch aus, diese kulturellen und religiösen Deutungsbemühungen wahrzunehmen, zu ordnen, in ein spannungsreiches Verhältnis zueinander zu setzen und Schülerinnen und Schüler zu befähigen, sich in diesem Geflecht konkurrierender Geltungsansprüche machtsensibel und machtkritisch zu positionieren.

5. Im Kontext der Corona-Krise wurde sowohl das Deutungsmuster bemüht, dass vor dem Virus alle Menschen gleich seien, als auch die verschärfte Wahrnehmung von sozialer Ungleichheit kommuniziert, weil keineswegs alle Milieus und Schichten gleichermaßen von dem Virus betroffen sind. Entsprechend waren auch die Bedingungen des Homeschooling keineswegs für alle gleich, sondern hinsichtlich des Gelingens u. a. sowohl von technischen Möglichkeiten als auch von der Betreuungsbereitschaft und -möglichkeit der Eltern und weiterer familiärer Bezugspersonen abhängig. So ist kommunikativ zu bearbeiten, welche verbindenden menschlichen Grunderfahrungen in dieser Krise sichtbar geworden sind und welche Unterschiede mit Blick auf die Dimensionen von Gerechtigkeit und Partizipationsmöglichkeiten kritisch zu reflektieren und nach Möglichkeit auszugleichen sind.

Der Religionsunterricht bietet Raum, um über Fragen von Gleichheit und Ungleichheit in der Krise und darüber hinaus kritisch nachzudenken.

6. Die Pandemie hat zahlreiche ethische Probleme aufgeworfen, die der gemeinsamen Reflexion und Bearbeitung bedürfen. Zum Beispiel: Wer verdient in der Gesellschaft Schutz? Wer wird möglicherweise auf wessen „Kosten“ geschützt? Nach welchen Kriterien soll entschieden werden? Wer wird gerettet, wenn nicht alle gerettet werden können? Wer wird zuerst und zu welchem Preis das „Anrecht“ auf eine Impfung erhalten? Auf der Metaebene betrachtet brechen damit bio-, medizin-, medien-, wirtschafts-, sozial- und religionsethische Fragen auf, die keinesfalls nur an ein bestimmtes Fach delegiert werden können, sondern in den verschiedensten Unterrichtsfächern Aufnahme verlangen. 
Der Religionsunterricht dient dazu, ethische Grundfragen zu formulieren, Entscheidungssituationen bewusst zu machen und Modelle zum Umgang mit den aktuellen Problemstellungen zu entwickeln.

7. In der Corona-Pandemie zeigt sich einmal mehr und mit besonderer Härte, dass sich mit der Welt auch die Risiken globalisiert haben. Zeitweilig waren mehr als 90\% aller Schülerinnen und Schüler weltweit von den Schulschließungen betroffen. Zugleich hat die Corona-Pandemie bei vielen Menschen das Gefühl verstärkt, Teil der einen, gefährdeten Menschheit zu sein. Dadurch haben universalisierende Diskurse an Bedeutung gewonnen: Es wird um das große Ganze gerungen, ja zum Teil vehement gestritten. Kurzum: Corona ist kein Problem einzelner Länder und Regionen; die Krise geht alle und jeden an - und kann folglich nur gemeinsam gelöst werden. Risikobewältigung in pandemischen Zeiten kann nur in internationaler und transnationaler Zusammenarbeit staatlicher und nichtstaatlicher Akteure gelingen - und setzt daher globale Solidarität voraus.

Der Religionsunterricht bringt in die Schule die Perspektive der Ökumene im Sinne der Einen bewohnten Erde ein und sorgt mit dafür, dass weltumspannende Vernetzungen der Menschheit im Blick sind und Bilder gelingenden Lebens im globalen Horizont entwickelt werden können.

8. Die Corona-Krise hat einen ungeheuren Digitalisierungsschub bewirkt, der dringend der grundlegenden Reflexion bedarf. Denn mit der Digitalisierung verbinden sich im Bildungskontext Fragen, die weit über die technische Ausstattung und die Versiertheit der Nutzerinnen und Nutzer hinausreichen. Zweifellos haben sich in der Krise durch die digitalen Medien an den Schulen neue Möglichkeiten der Kontaktaufnahme und des Fernunterrichts ergeben. Dabei gilt es aber auch zu klären, wie weit mitmenschliche Verbundenheit im digitalen Raum reicht und wo sie ihre Grenze hat. $\mathrm{Zu}$ bedenken ist, was humane Kommunikation in ihrer Fülle und Leiblichkeit ausmacht und welche religiösen Kommunikationsprozesse sich sinnvollerweise in digitale Räume übertragen lassen und welche nicht.

Der Religionsunterricht fördert digitales Lernen - aber nicht nur das: Darüber hinaus reflektiert er die Chancen und Grenzen digitaler Kommunikation im Blick auf ihre Relevanz und fragt nach der Qualität mitmenschlicher Verbundenheit angesichts der aktuellen Krise und darüber hinaus. 
9. Die im Zuge der Pandemie aufgebrochene Frage nach dem Wohl der Menschheit als Ganzer wird im christlichen Glauben von der hoffnungsstarken Perspektive des Reiches Gottes bestimmt und in Bibel und Theologie konkretisiert. Dabei können verschiedene biblische Deutungsangebote und Sprachformen kritisch aufgenommen und auf ihre Tragfähigkeit in der Krise hin befragt werden - zu denken ist hier etwa an die Psalmen, Hiob oder die Wunder- und Heilungserzählungen sowie an die Passion und Auferstehungsverheißung.

Der Religionsunterricht ist ein unverzichtbarer Baustein dafür, dass Kinder und Jugendliche Schule unter Bezugnahme auf christliche und andere religiöse Deutungsangebote als Ort der Verantwortung und Gestaltung gemeinsamen Lebens wahrnehmen.

10. Leben und Überleben in Krisen kann nur gelingen, wo sich am Ende des Tunnels ein Lichtschein abzeichnet. Wo nur noch alles dunkel ist, wo kein Hoffnungslicht mehr durchscheint, da sind die Ressourcen auch der Krisenbewältigung rasch erschöpft. Visionen für das Leben nach der Krise, wie sie aus dem christlichen Glauben auch angesichts der Krise erwachsen können, sind hier neu gefragt. Dies wirft in bildungspolitischer Hinsicht erneut die Frage nach dem Zusammenspiel der einzelnen Fächer sowie nach dem Selbstverständnis der Lehrkräfte und der Orientierungsfunktion von Schule in der Krise auf.

Der Religionsunterricht zeichnet sich dadurch aus, dass seine Lehrkräfte Formen kompetenter theologischer und sensibler Begleitung anbieten und erkennen, wo weiterführende schulseelsorgerliche oder therapeutische Hilfe für die Einzelnen notwendig und wie zugleich die Pflege einer gemeinsamen Schulkultur in Zeiten der Krise möglich ist.

Frankfurt am Main/Jena/Rostock/Zürich/Tübingen/Berlin, den 3. September 2020

David Käbisch, Ralf Koerrenz, Martina Kumlehn, Thomas Schlag, Friedrich Schweitzer und Henrik Simojoki (als Herausgeber/in der Zeitschrift für Pädagogik und Theologie) 